\title{
Coleta de amostras e métodos analíticos para determinação de chumbo
}

\section{Collection of samples and analytical methods for lead determination}

\author{
Márcia Andreia Mesquita Silva da Veiga
}

\begin{abstract}
RESUMO
Chumbo é, frequentemente, usado como um indicador de poluição. Uma vez liberado no meio ambiente, este poluente pode seguir diferentes rotas, podendo ser convertido em espécies mais solúveis, tornado-se biodisponível. Neste artigo é apresentado de forma sucinta chumbo como poluente urbano, procedimentos de amostragem (coleta de amostras), transporte, armazenamento e preservação de amostras de interesse ambiental (solos e águas) e amostras clínicas (sangue e urina). A importância e classificação das Salas Limpas, assim como as principais técnicas analíticas para a determinação de chumbo - AAS, ICP OES e ICP-MS - também são abordadas.
\end{abstract}

Palavras-Chave: Determinação/Chumbo. Salas Limpas. Espectrofotometria Atômica/ Técnicas.

\section{Chumbo: poluente urbano}

Em grandes metrópoles, como a cidade de São Paulo, o desenvolvimento econômico e o crescimento populacional, permitiram que a poluição, causada principalmente por automóveis e indústrias, atingisse níveis críticos. Devido a estas atividades, a concentração de metais tóxicos, como chumbo, é alta. É possível observar, ao sobrevoar grandes metrópoles, uma nuvem cinza, espessa e densa pairando sobre a cidade. São toneladas de substâncias tóxicas que fazem parte da composição do ar, de origem antropogênica. Chumboé, frequentemente, usado como um indicador de poluição. Uma vez liberado no meio ambiente, este poluente pode seguir diferentes rotas, podendo ser convertido em espécies mais solúveis, tornado-se biodisponível para os seres vivos. A identificação das formas químicas e distribuição dos metais tóxicos que são liberados, aprisionados ou adsorvidos nas partículas dos solos, é fundamental para entender suas características químicas e físicas no ambiente, pois são essas características que controlam a mobilidade e biodisponibilidade.
1. Docente. Departamento de Química, Faculdade de Filosofia, Ciências e Letras de Ribeirão Preto - USP
Correspondência:

Profa. Dra. Márcia Andreia Mesquita Silva da Veiga Departamento de Química, Faculdade de Filosofia, Ciências e Letras de Ribeirão Preto, Universidade de São Paulo Av. Bandeirantes, 300 Bairro Monte Alegre 14040-901 - Ribeirão Preto, SP eMAIL: mamsveiga@ffclrp.usp.br

Artigo recebido em 05/10/2009 Aprovado em 08/12/2009 
Chumbo ocorre nos solos como uma mistura de compostos químicos na fase sólida, variando na morfologia e no tamanho da partícula. Estes componentes incluem fases minerais discretas, espécies coprecipitadas e adsorvidas associadas com minerais do solo ou matéria orgânica, e espécie dissolvidas que podem estar complexadas com uma variedade de ligantes orgânicos e inorgânicos. A ocorrência e a distribuição relativa de um elemento entre estas várias fases e o solo, irão controlar as propriedades de dissolução de um elemento e, em consequência, sua biodisponibilidade. Com o tempo, ocorrem mudanças na distribuição de um elemento entre estas várias fases, como resultado de desgaste químico e físico, processos biológicos, infiltração da água e distúrbios antropogênicos, mudando a biodisponibilidade deste elemento. Por exemplo, se acetato de chumbo, que é muito solúvel, for liberado no solo, o chumbo rapidamente irá se incorporar aos minerais dos solos, e sua biodisponibilidade diminuirá com o tempo. Em contraste, poucas mudanças podem acontecer na biodisponibilidade do chumbo disperso em resíduos de sílica fundida (devido a sua relativa insolubilidade), mesmo depois de décadas no meio ambiente. ${ }^{1}$

Um dos maiores problemas ambientais deste ramo de ciência é o chamado passivo ambiental, herança da desativação de empreendimentos industriais. Em termos gerais, a contaminação dos solos e das águas subterrâneas é dos mais graves passivos ambientais. $\mathrm{O}$ conceito de passivo ambiental é utilizado para descrever o acúmulo de danos ambientais ocasionados pelo fechamento de instalações industriais que devem ser reparados a fim de que seja mantida a qualidade ambiental de um determinado local. Os solos contaminados podem apresentar risco à saúde pública de várias formas: por contato direto com a pele, por ingestão ou inaladas ao se fixar a substâncias sólidas, ou por causa de emissão de gases tóxicos; também podem ser transferidas para as águas subterrâneas, contaminando redes de água potável, ou contaminando cursos de água superficiais, sendo também tóxicos para outros seres vivos do ecossistema. ${ }^{2} \mathrm{De}$ um modo geral, a contaminação por exposição direta se dá através da ingestão de solos, absorção dérmica, inalação de gases e inalação de partículas; a contaminação por exposição indireta se dá por ingestão de vegetais contaminados e ingestão de água (tratada ou não).

Diante do exposto nos parágrafos acima, a coleta do material clínico a ser analisado, assim como os procedimentos analíticos que serão adotados na determinação de chumbo, desempenham um papel fundamental, pois vivemos em um ambiente notadamente poluído com chumbo.

\section{Coleta de amostras}

\section{Ambientais: águas e solos}

Para coleta de amostras de águas naturais, a quantidade mínima requerida é de $1 \mathrm{~L}$, de acordo com o Standard Methods for the Examination of Water and Wastewater. ${ }^{3} \mathrm{O}$ recipiente de coleta pode ser de plástico (polietileno ou equivalente) ou de vidro, previamente lavados com solução $1+1$ de $\mathrm{HNO}_{3}$. A conservação é feita através da adição de $\mathrm{HNO}_{3}$ até $\mathrm{pH}=2$; se a intenção é medir chumbo dissolvido, a amostra deve ser filtrada imediatamente, seguido da adição do ácido nítrico. Nestas condições estabelecidas, a amostra pode ser armazenada por até 6 meses antes da análise que geralmente é realizada por espectrometria de absorção atômica com atomização eletrotérmica (ET AAS, do inglês, electrothermal atomic absorption spectrometry) ou por espectrometria de massa com fonte de plasma indutivamente acoplado (ICP-MS, do inglês inductively coupled plasma mass spectrometry).

Quando se tratar de coleta de amostras de águas em mananciais, a coleta deve ser realizada no sentido contrário da correnteza, com profundidade mínima de $20 \mathrm{~cm}$. Para poços, o frasco de coleta deve descer preso a um cordão, permitindo que o mesmo seja submergido até o máximo de profundidade. Não permitir que, no momento da descida, o frasco toque nos lados do poço.

A coleta de águas em torneiras, de acordo com a Organização Panamericana de Saúde4, deve ser precedida por uma limpeza das partes internas e externas da torneira com álcool, após sua abertura por alguns segundos. Após este procedimento, a torneira deve ser aberta novamente e permitido escoar a água por mais alguns segundos. Em seguida, a coleta pode ser realizada em frasco previamente descontaminado (solução $1+1 \mathrm{HNO}_{3}$ ) em um nível logo acima da metade do frasco.

Para solos, a coleta deve proceder de acordo com o objetivo pretendido da análise. Geralmente, se a finalidade é monitoramento ou screening, a amostra é coletada no horizonte A $(0-20 \mathrm{~cm})$. Após a coleta, as amostras deverão ser secas, destorradas e penei- 
radas em malha com abertura de $2 \mathrm{~mm}$ para obtenção de terra fina seca ao ar. $\mathrm{O}$ armazenamento deve ser em refrigeração. Essas amostras devem ser as mais representativas possíveis do material original ou área a ser estudada.

A amostragem é um ponto importante, pois pode ser realizada de três maneiras: direcionada, sistemática e aleatória. De acordo com a norma ISO/DIS 10381-1, na amostragem direcionada a locação dos pontos de amostragem é realizada de acordo com o conhecimento já existente a respeito das fontes e vias de disseminação da contaminação, evidências visuais de contaminação do solo ou aplicação de métodos de screening na área. ${ }^{5}$ Uma forma muito comum de distribuição aleatória dos pontos de amostragem é o estratificado (ISO/DIS 10381-1), em que se procede a uma divisão regular da área, distribuindo-se os pontos de forma aleatória em cada uma delas. Com esse esquema de amostragem, assegura-se que todas as subáreas que compõe o local investigado serão amostradas, o que pode não ocorrer em um esquema aleatório simples. ${ }^{5}$ Uma forma de contornar os problemas da amostragem aleatória estratificada é o emprego de um esquema com distribuição sistemática dos pontos de amostragem (malha de amostragem) que, além de evitar a coleta de amostras em pontos muito próximos, apresenta as mesmas vantagens da subdivisão da área. Segundo a USEPA (do inglês, United States Environmental Protection Agency), a experiência e as considerações teóricas mostram que na maioria dos casos a aplicação de uma malha regular com distribuição sistemática dos pontos de amostragem mais práticos gera um retrato detalhado da variação das propriedades do solo existentes no local. Tem como vantagem a facilidade de implantação no campo e a possibilidade de adensamento do número de pontos em que for necessário, por meio de uma amostragem direcionada. $^{5}$

Para a coleta de solos superficiais os equipamentos de amostragem mais comuns são: pás; trados de caneco manuais ou mecânicos; trado de rosca; trado holandês; amostradores tubulares (barrilete "tubo aberto", meia-cana, tubo fechado e tubo de parede fina).

\section{Clínicas: sangue e urina}

Para a determinação de chumbo em sangue total, a quantidade de sangue necessária pode variar de acordo com o laboratório - de 5 a $20 \mathrm{~mL}$, adicionados de $200 \mathrm{~L}$ de heparina. O horário da coleta é flexí- vel, desde que tenha ocorrido exposição há pelo menos cinco dias. O recipiente usado na coleta são tubos específicos (Vacutainer ${ }^{\circledR}$ ) livre de metais. Se a coleta for realizada longe do laboratório de análise, o transporte deve ser feito sob refrigeração a $4^{\circ} \mathrm{C}$, e o tempo entre a coleta e o envio da amostra ao laboratório não deve exceder 2 dias. Uma vez no laboratório de análise, o armazenamento destas amostras deve ser em geladeiras com temperatura entre 2 e $8^{\circ} \mathrm{C}$, por um período máximo de 5 dias. A determinação, geralmente, é realizada por ET AAS ou ICP-MS. ${ }^{6,7}$

É importante ter sempre em mente que não apenas o tubo, utilizado para coleta de amostra (Vacutainer®) deve ter quantidade mínima de chumbo, e o local da punção (no caso de sangue) devem ser limpos previamente à coleta, mas toda a manipulação pré-analítica deve ser feita em local adequado, com ponteiras, tubos e reagentes com quantidades muito baixas de metais. Para limpeza adequada para medir chumbo, tubos e ponteiras novos precisam ser submersos em ácido nítrico (20-50\%) 18 horas, secos a seguir em capelas de fluxo laminar (Classe 100), e depois guardados hermeticamente até o momento do uso. Normalmente é mais vantajoso comprar tubos para as diluições prévias à análise também já livres de metais, pois há sempre risco de contaminação aleatória durante esse procedimento de limpeza dos tubos e o custo é alto para essa limpeza. Outra questão a considerar é o risco de acidentes durante a manipulação de ácido nítrico. Os reagentes também precisam ser de qualidade ultrapura (por ex. os da linha Suprapure da Merck), ou então, no caso dos ácidos, devem ser bidestilados em laboratórios preparados para este procedimento e armazenados em frascos descontaminados para metais. Reagentes ultrapuros devem ser usados em conjunto com água deionizada 18-25 Mohm.cm para limpeza final e digestão das amostras. Brancos também devem ser preparados para cada conjunto de amostras.

Geralmente, a determinação de chumbo em amostras de urina é realizada para monitorar a quantidade de chumbo excretado pelo paciente, durante o tratamento de quelação. A quantidade de urina coletada também pode variar de 20 a $50 \mathrm{~mL}$, durante 24 horas. O recipiente para coleta pode ser de plástico e o transporte, caso a coleta não seja realizado no próprio laboratório de análises clínicas, deve acontecer sob refrigeração a $4^{\circ} \mathrm{C}$, em um período de tempo que não exceda um dia - o ideal é que seja imediatamente. No laboratório de análises o armazenamento deve ser 
em geladeira com temperatura controlada entre $2 \mathrm{e}$ $8^{\circ} \mathrm{C}$, por no máximo 3 dias. As técnicas que figuram para seu monitoramente são: espectrofotometria de absorção molecular no ultravioleta e visível (UV/Vis) para detecção da creatinina, ET AAS ou ICP-MS para detecção direta do chumbo. É importante ressaltar que, muitas vezes, é necessário realizar uma digestão ácida nas amostras clínicas, antes de analisá-las diretamente por ET AAS ou ICP-MS. ${ }^{6,7}$

\section{Salas Limpas}

De acordo com a NBR 13413 (NBR 13700 Normas de Salas Limpas), sala limpa é uma sala na qual o suprimento e a distribuição do ar, filtragem, materiais de construção e procedimentos de operação, visam controlar as concentrações de partículas em suspensão no ar, atendendo aos níveis apropriados de limpeza, conforme definidos pelo usuário e de acordo com as normas técnicas vigentes. ${ }^{8}$

As salas limpas são classificadas pela pureza de seu ar. O método mais facilmente conhecido e universalmente aplicado e é sugerido pela Federal Standard 209, em que o número de partículas igual ou maior que $0,5 \mu$ m são medidas em um pé cúbico e esta contagem é usada para classificar a sala. ${ }^{9}$ de alta qualidade, na montagem e teste de giroscópios de precisão, e na montagem de mancais miniaturizados. Salas Classe 10.000 são empregadas na montagem equipamentos hidráulicos e pneumáticos de precisão, válvulas servo-controladas, dispositivos de relógios de precisão, e engrenamento de alto grau. Por fim, Salas Classe 100.000 servem para trabalhos óticos em geral, montagem de componentes eletroeletrônicos, montagem hidráulica e pneumática.

Existem dois tipos de salas limpas: as de fluxo convencional e unidirecional. Salas Limpas de fluxo convencional são também conhecidas com Salas Limpas de ventilação turbulenta ou não unidirecional. Sala Limpas de fluxo unidirecional são conhecidas como de fluxo laminar ou salas ultralimpas.

A importância em usar estes tipos específicos de salas reside no impacto da pesquisa desenvolvida, ou seja, em questões de saúde pública, legais e forenses, a exatidão e precisão dos resultados podem nortear políticas ambientais e de saúde, assim como um veredito. Se determinações na ordem de partes por bilhão ou partes por trilhão são requeridas, o uso de Salas Limpas é uma necessidade. Não podemos esquecer também que, a obrigatoriedade de vestimentas adequadas nestes ambientes é um ponto crítico, pois a

\begin{tabular}{|lllllll}
\hline Classificação Federal Standard 209 & 1 & 10 & 100 & 1000 & 10000 & 100000 \\
\hline No de partículas por pé cúbico com tamanho $\geq 0,5 \mu \mathrm{m} .^{3}$ & 1 & 10 & 100 & 1000 & 10000 & 100000
\end{tabular}

Essa classificação atende diferentes atividades. Uma sala classe 1 é usada somente para manufatura de circuitos integrados com desenvolvimento de geometrias sub-mícron. Salas Classe 10 são usadas para manufatura de semicondutores produzidos em larga escala, circuitos integrados com linhas menores que 2 micras. Salas Classe 100 são usadas quando se deseja ambientes livres de partículas (neste tipo Sala se enquadra as determinações clínicas de $\mathrm{Pb}$ em material biológico) e bactérias. É empregada também na manufatura de produtos médicos injetáveis, em operações de implantes ou transplantes cirúrgicos, na fabricação de circuitos integrados e no isolamento de pacientes imonudeprimidos e pacientes com operações ortopédicas. Salas Classe 1.000 também são empregadas em determinadas análises inorgânicas, mas são principalmente usadas na fabricação de equipamento quantidade microorganismos carregados pelo homem na pele da cabeça, por exemplo, é de $1,5 \times 10^{4} \mathrm{UFC} /$ $\mathrm{cm}^{2}$ (UFC = unidade formadora de colônia); no antebraço é de $1,0 \times 10^{3} \mathrm{UFC} / \mathrm{cm}^{2}$; nas mãos e dedos: 1,0 x $10^{2} \mathrm{UFC} / \mathrm{cm}^{2}$; secreção do nariz: $10^{7} \mathrm{UFC} / \mathrm{g}$, no ato de espirrar: $10^{8} \mathrm{UFC}$, no cerume dos ouvidos: $10^{6} \mathrm{UFC}$, na saliva: $10^{7} \mathrm{UFC} / \mathrm{mL}$ e nas fezes $10: 10^{11} \mathrm{UFC} / \mathrm{g}$.

\section{Principais Técnicas Analíticas}

\section{Espectrometria de Massa com Fonte de Plas- ma Indutivamente Acoplado}

O desenvolvimento do espectrômetro de massa com fonte de plasma indutivamente acoplado, ICPMS (do inglês - inductively coupled plasma mass spectrometry) foi induzido em parte pela sua aplica- 
ção nas ciências da terra. A análise de amostras geológicas consideradas uma das mais difíceis aplicações espectroquímicas, requer medidas exatas e precisas, como por exemplo, na datação e caracterização de rochas e minérios. Devido ao seu potencial, ICP-MS é uma das principais técnicas analíticas para a análise na geoquímica, além da sua aplicação na área ambiental, clínica e alimentos; e isto se deve aos baixos limites de detecção (LD's) possíveis, ampla faixa dinâmica linear, rápida determinação das razões isotópicas, pequeno volume de amostra, além, é óbvio, da sua capacidade multielementar. Além disso, esta técnica é capaz de realizar uma análise semi-quantitativa, em menos de 1 minuto. A espectrometria de massa (MS) proporcionou benefícios significativos para a espectroscopia ICP. Talvez o benefício que recebeu a maior parte da atenção é a capacidade de atingir baixos limites de detecção - cerca de $90 \%$ dos elementos podem ser determinados com LD's na faixa de 0,1 a $0,001 \mathrm{ng} / \mathrm{mL}$ em soluções aquosas. ${ }^{11-12}$

A configuração do instrumento consiste em três componentes principais: (1) um ICP de argônio convencional que opera a temperaturas de 6000 a 10000 $\mathrm{K}$, (2) um espectrômetro de quadrupolo convencional, e (3) uma interface. Nestas temperaturas ( 8000 K), possíveis interferências de substâncias químicas são insignificantes (para soluções inorgânicas), e essencialmente todos os átomos são ionizados em uma larga extensão. ${ }^{11-12}$

\section{Espectrometria de Emissão Óptica com Fon- te de Plasma Indutivamente Acoplado}

Embora este técnica não possua a mesma sensibilidade instrumental da ICP-MS, ela configura como uma técnica bem estabelecida, capaz de fornecer análises quantitativas multielementares, com ampla faixa dinâmica de trabalho (entre ppb e ppm), para elementos com comprimentos de onda compreendidos entre 190 a $800 \mathrm{~nm}$. Seu princípio se baseia no fato de que um átomo livre, no estado fundamental, pode receber energia suficiente para que seus elétrons na camada de valência comecem a sofrer transições. Estes elétrons saltam para camadas mais externas, permanecendo por um tempo muito curto; por ser um estado pouco estável quando retornam para a camada de menor energia, emitem um fóton em $\lambda$ específico. Cada elemento possui seu próprio conjunto característico de níveis de energia e assim, seu próprio e úni- co conjunto de comprimento de onda de emissão e absorção.

A instrumentação consiste em um ICP de argônio, a partir do qual serão emitidos os comprimentos de onda dos analitos desejados, um espectrômetro, onde ocorrerá a dispersão e separação do feixe de luz. Um dos detectores mais modernos para este tipo de equipamento é do estado sólido, conhecido como CCD (charge-coupled device).

\section{Espectrometria de Absorção Atômica}

O princípio fundamental da AAS envolve a medida da absorção da intensidade de radiação eletromagnética, proveniente de uma fonte de luz, por átomos gasosos no estado fundamental. Com base neste princípio, o elemento de interesse (metal, semi-metal e não-metal), no estado vapor, absorve a radiação de um comprimento de onda específico pela transição de seus elétrons para um nível mais energético. Devido a isso, a AAS torna-se uma técnica específica, pois se utiliza de uma fonte capaz de emitir uma radiação de comprimento de onda específico para que ocorra a transição eletrônica característica do elemento de interesse. Este princípio teve origem em 1860, com o físico Kirchhoff que estabeleceu a lei geral da Absorção e Emissão da Energia pela matéria. "Todos os corpos podem absorver a radiação que eles próprios emitem". Em 1955, Alan Walsh descobriu que as maiorias dos átomos livres na chama se encontravam no estado fundamental (99\%) e os restantes (1\%) ionizados ou excitados. Com isso, foi possível desenvolver o primeiro equipamento de absorção atômica.

Os principais componentes de um espectrômetro de absorção atômica são: uma fonte de luz primária, sistema de atomização (chama e forno de grafite), um monocromador para isolar o comprimento de onda específico a ser medido, um detector para medir com exatidão.

Esta técnica configura-se como uma das técnicas atômicas mais robustas, confiáveis e bem estabelecidas. Embora seja monoelementar, é capaz de apresentar limites de detecção comparáveis aos da ICPMS, quando usada no modo forno de grafite (ETAAS), o qual é em 3 ordens de magnitude mais sensível do que a chama (F AAS). A figura 1 mostra de maneira simplificada, os limites de detecção das três técnicas discutidas. Na figura 2 temos os custos envolvidos na aquisição de cada equipamento. 


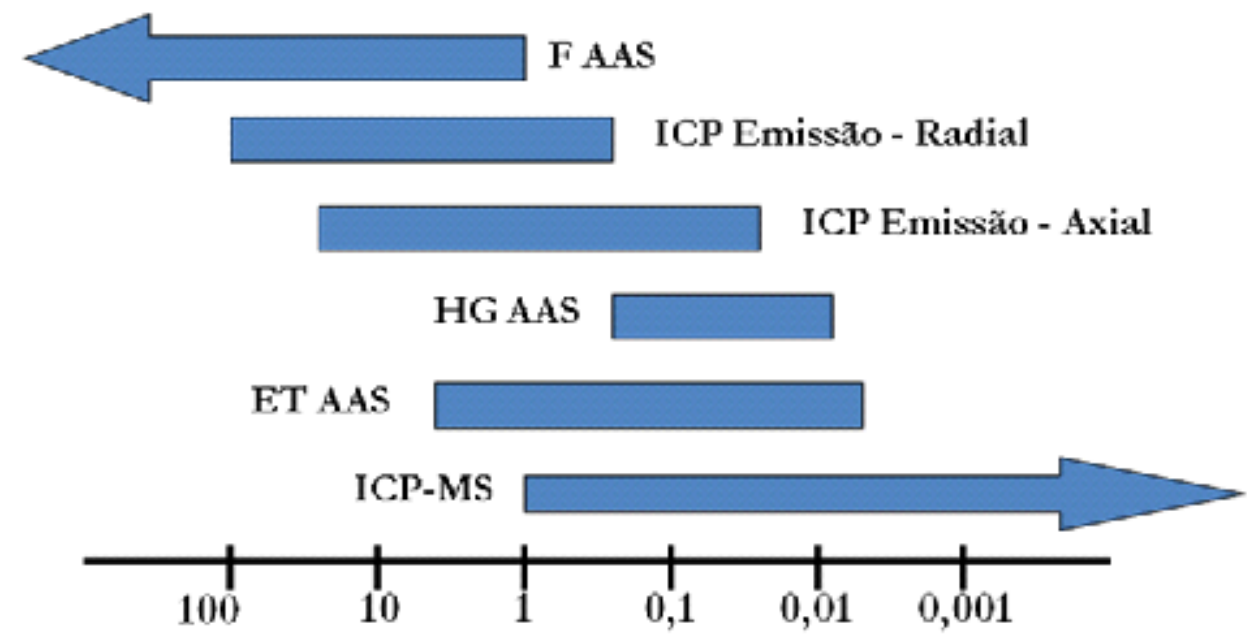

Faixa L.O.D. (ppb ou $\mu \mathrm{g} / \mathrm{L}$ )

Figura 1. Limites de deteç̧ão para as principais técnicas espectroscópicas atômicas (Adaptado de 13).

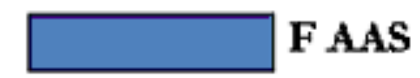

\section{ET AAS}

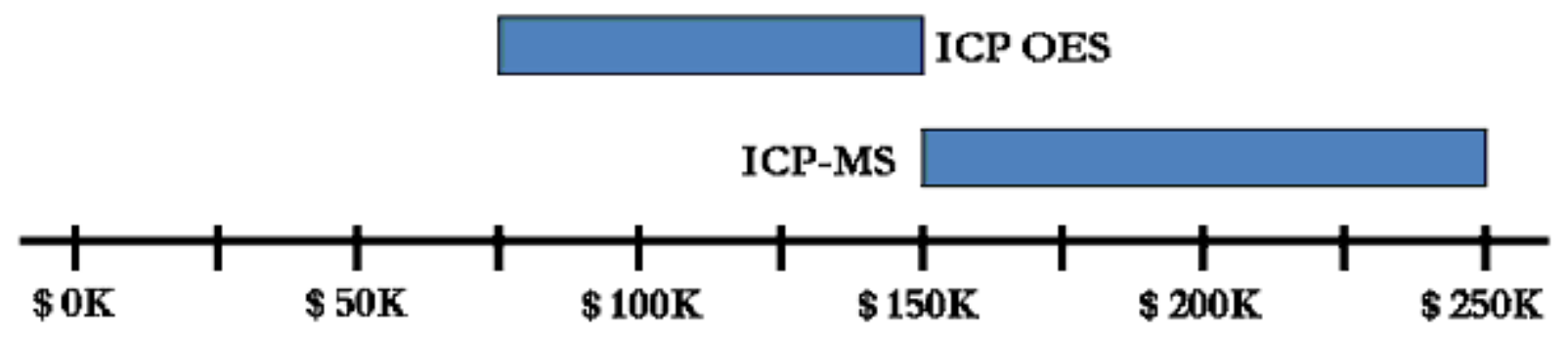

Figura 2. Custos relativos para sistemas de espectroscopia atômica (valores em dólares). Adaptado de 13.

\section{ABSTRACT}

The metal lead has frequently used as a sensitive pollution indicator. Once released in the environment, this pollutant can follow different ways, being converted in highly soluble species, became bioavailable. In this paper is presented in a succinct form, lead as urban pollutant, sampling procedures (collection of samples), transport, storage and preservation of environmental (soils and waters) and clinical (blood and urine) samples. The importance and classification of clean rooms, as well as the main analytical techniques for lead determination - AAS, ICP-OES and ICP-MS - are broached too.

Keywords: Lead/Determination. Clean Rooms. Spectroscopy Atomic/Techniques. 


\section{Referências bibliográficas}

1. Ruby MV, Schoof R, Brattin W, Goldade M, Post G, M . Harnois et al. Advances in evaluating the oral biodisponibility of inorganic in soil for use in human health risk assessment, Environ sci technol. 1999; 33: 3697-705.

2. Sánchez LE. Desengenharia. O passivo ambiental na desativação de empreendimentos industriais. São Paulo: Edusp; 2001.

3. American Public Health Association; American Water Works Association;Water Environment Federation. Standard Methods for the Examination of Water and Wastewater. 21st Edition, 2005.

4. Organização Pan Americana de Saúde (OPAS) (apud Manual de Coleta de Amostras, Fundação Ezequiel dias, 2009).

5. CETESB - Companhia de Tecnologia de Saneamento Ambiental. Manual CETESB 6300 amostragem do solo.

6. Brasil. Ministério da Saúde. Atenção à saúde dos trabalhadores expostos ao chumbo metálico. Ministério da Saúde, Secretaria de Atenção à Saúde, Departamento de Ações Programáticas Estratégicas. Brasília: Editora do Ministério da Saúde, 2006. (Série A. Normas e Manuais Técnicos).
7. Manual de Coleta de Amostras. Fundação Ezequiel Dias, Instituto Octávio Magalhães, Divisão de Vigilância Sanitária, Belo Horizonte: 2009.

8. Associação Brasileira de Normas Técnicas (ABNT). NBR 13700 - Normas de Salas Limpas.

9. Federal Standard 209E (www.iest.org/publctns/ fedstd209.htm) [Acessado em 18/09/2009].

10. PDA - International Association for Pharmaceutical Science and Technology (www.pda.org) [Acessado em 18/09/2009].

11. Houk RS, Jarvis KE, Gray AL. Handbook of Inductively Coupled Plasma Mass Spectrometry, Chapman and Hall, New York: 1992.

12. Veiga MAMS. Determinação de elementos traço por AAS e ICP-MS após extração no ponto nuvem, usando dietilditiofosfato como agente quelante. [Tese de Doutorado] Programa de Pós-Graduação em Química da Universidade Federal de Santa Catarina, 2000.

13. Guide to Inorganic Analysis. Perkin Elmer Life and Analytical Sciences. www.perkinelmer.com [Acessado em 18/09/ 2009]. 